\title{
Embedding method for confined quantum systems
}

\author{
S. Crampin* \\ Cavendish Laboratory, Madingley Road, Cambridge CBз OHE, United Kingdom \\ M. Nekovee and J.E. Inglesfield \\ Institute for Theoretical Physics, University of Nijmegen, NL-6525 ED Nijmegen, The Netherlands
}

(Received 25 October 1994)

\begin{abstract}
We discuss the application of the embedding method to the problem of finding the eigenstates of confined quantum systems. Embedding is a general way of tackling boundary condition problems, giving a true variational principle, and we apply it to the confinement problem by embedding within an isotropic medium with a very large potential. Corrections for incomplete confinement are described. The method is tested on examples recently studied by Brownstein [Phys. Rev. Lett. 71, 1427 (1993)], namely, an electron in two dimensions confined within the quadrant of a circle, and a $\mathrm{H}$ atom off center in a spherical cavity.
\end{abstract}

In this paper, we show how the embedding method, ${ }^{1}$ a variational method for taking care of boundary conditions on the wave function, can be used to find the eigenstates of quantum systems confined by an effectively infinite potential barrier. There have been several papers recently on this type of problem, in particular, for solving the Schrödinger equation for a $\mathrm{H}$ atom confined in cylindrical $^{2-4}$ and spherical ${ }^{5-7}$ cavities. These have used trial wave functions vanishing on the boundary walls (the requirement on the exact solution of the problem),$^{2-5}$ nonvanishing basis functions with constraints that the trial function vanishes at a finite set of points, ${ }^{6}$ and a stationary principle due to Brownstein ${ }^{7}$ for a trial function not necessarily vanishing on the boundary. The embedding method can tackle this class of problem, and it gives a minimum variational principle. Advances in nanostructure fabrication techniques mean that this is not of purely theoretical interest-for example, the cylindrical confinement problem is relevant to an impurity atom in a quantum wire ${ }^{4}$ and the spherical case to an impurity in a quantum dot. 8

In the embedding method, ${ }^{1}$ we consider the region of interest I joined on to region II, and derive a variational principle for a trial function $\phi$ defined explicitly only in region I-the boundary condition that the wave function must be joined on to the solution of the Schrödinger equation in region II is replaced by additional boundary terms in the Hamiltonian for region I. The original motivation for this approach was to develop a method for solving the Schrödinger equation in a defect region of a solid, using basis functions of finite extent in the defect region (I) and the embedding potential taking care of the infinitely extended substrate (II) (a recent application is to adsorbates ${ }^{9}$ ). Here region $I$ is the cavity, and the very high potential beyond the boundary of the cavity constitutes region II. The variational principle is derived by notionally extending $\phi$ into II with an exact solution of the Schrödinger equation at some energy $\epsilon$ (in fact a parameter), which matches in amplitude to $\phi$ over the boundary surface $S$, separating I and II. The contribution of the wave function in II to the expectation value of the Hamiltonian is then eliminated using Green's theorem. ${ }^{1}$ Using Hartree atomic units, with $e=\hbar=m=1$, the resulting expression for the expectation value is

$$
E=\frac{\int_{\mathrm{I}} d^{3} r \phi H \phi+\int_{S} d^{2} r_{S} \frac{1}{2} \phi \frac{\partial \phi}{\partial n_{S}}+\int_{S} d^{2} r_{S} \int_{S} d^{2} r_{S}^{\prime} \phi\left(G_{0}^{-1}-\epsilon \frac{\partial G_{0}^{-1}}{\partial \epsilon}\right) \phi}{\int_{\mathrm{I}} d^{3} r \phi^{2}-\int_{S} d^{2} r_{S} \int_{S} d^{2} r_{S}^{\prime} \phi \frac{\partial G_{0}^{-1}}{\partial \epsilon} \phi}
$$

The first integral in the numerator is the expectation value of the Hamiltonian through region $I$, the region of interest; the second is an integral over the boundary surface $S$ involving the normal derivative of the trial function - combined with the kinetic energy operator in $H$, the surface normal derivative ensures a Hermitian operator for integrals restricted to I; and the third term is a double integral over $S$, involving the surface inverse of the Green function $G_{0}$ for region II evaluated at energy $\epsilon$ with zero normal derivative on $S . G_{0}^{-1}$ is the embedding potential, and it ensures that when the energy is mini- mized, $\phi$ not only satisfies the Schrödinger equation in I, but also matches in amplitude and derivative, the exact solution in II. The energy derivative of $G_{0}^{-1}$, which appears in both the numerator and denominator gives the normalization of $\phi$ in II, and provides a correction to the embedding potential so that it is evaluated (to first order) at the energy $E$ rather than the trial energy $\epsilon$.

The embedding potential cannot be defined for an infinite potential in II, and so to apply this method to confined systems, we choose a constant but very large potential $V$ in II. As a result, the variational principle will con- 
verge from above to the lowest eigenvalue of this system, but this will, in principle, lie below the true eigenvalue of the actual confined system, as there is slight leakage of the wave function out of I. We discuss below how this error may be assessed. The large potential leads to a great simplification in the embedding formalism. Let us consider, for example, confinement in a spherical cavity of radius $R$, for which the embedding potential can be expanded as a sum over spherical harmonics:

$$
G_{0}^{-1}\left(\mathbf{r}_{S}, \mathbf{r}_{S}^{\prime}\right)=\sum_{L} \mathcal{G}_{L} Y_{L}(\Omega) Y_{L}^{*}\left(\Omega^{\prime}\right)
$$

For large $V, \mathcal{G}_{L}$ is given by

$$
\mathcal{G}_{L}=\frac{\sqrt{2 V}}{2 R^{2}}\left[1+O\left(\frac{\epsilon}{V}\right)\right]+O(1)
$$

so

$$
G_{0}^{-1}\left(\mathbf{r}_{S}, \mathbf{r}_{S}^{\prime}\right) \approx \sqrt{\frac{V}{2}} \delta\left(\mathbf{r}_{S}-\mathbf{r}_{S}^{\prime}\right)
$$

Because $\partial G_{0}^{-1} / \partial \epsilon$ is negligible compared with $G_{0}^{-1}$ for large $V$, the variational expression (1) then simplifies to

$$
E=\frac{\int_{\mathrm{I}} d^{3} r \phi H \phi+\int_{S} d^{2} r_{S}\left\{\frac{1}{2} \phi \frac{\partial \phi}{\partial n_{S}}+\sqrt{\frac{V}{2}} \phi^{2}\right\}}{\int_{\mathrm{I}} d^{3} r \phi^{2}} .
$$

This holds for a cavity of arbitrary shape, as minimizing $E$ leads to a wave function $\phi$ not only satisfying the Schrödinger equation within I, but also satisfying

$$
\frac{\partial \phi}{\partial n_{S}}=-\sqrt{2 V} \phi
$$

over $S$, which for large $V$ and well-behaved functions means

$$
\phi\left(\mathbf{r}_{S}\right) \approx 0
$$

as we require.

In practice very large values of $V$ can be used, so the error in the eigenvalue due to leakage can be made as small as we require. Furthermore, the error varies approximately as $1 / \sqrt{V}$, and so extrapolation of the eigenvalue to complete confinement can be made. To show this behavior, we consider $\psi$ - the solution we requirewhich satisfies the Schrödinger equation in I with zero amplitude boundary condition on $S$ at energy $E_{0}$. Then, from Green's theorem, the difference between $E_{0}$ and $E$, the energy of $\phi$ satisfying the Schrödinger equation with boundary condition (6), is given by

$$
E_{0}-E=\frac{-\frac{1}{2} \int_{S} d^{2} r_{S} \phi \frac{\partial \psi}{\partial n_{S}}}{\int_{\mathrm{I}} d^{3} r \phi \psi}
$$

and using (6), this becomes

$$
\begin{aligned}
E_{0}-E & =\frac{1}{2 \sqrt{2 V}} \frac{\int_{S} d^{2} r_{S} \frac{\partial \phi}{\partial n_{S}} \cdot \frac{\partial \psi}{\partial n_{S}}}{\int_{\mathrm{I}} d^{3} r \phi \psi} \\
& \approx \frac{1}{2 \sqrt{2 V}} \int_{S} d^{2} r_{S}\left(\frac{\partial \psi}{\partial n_{S}}\right)^{2}
\end{aligned}
$$

(assuming normalized wave functions). Hence we obtain a $1 / \sqrt{V}$ variation, and knowing this error behavior removes the apparent drawback of using a finite $V$.

As a first example we consider the same model problem as Brownstein, ${ }^{7}$ a free electron in two dimensions confined within a quadrant of a circle with radius $R$. The exact solutions of this problem have the form (using cylindrical polar coordinates)

$$
\phi_{p, k}(\rho, \theta)=J_{p}\left(\lambda_{p, k} \rho / R\right) \sin (p \theta),
$$

where $p$ is an even integer, $J_{p}$ is a Bessel function, and $J_{p}\left(\lambda_{p, k}\right)=0$. The corresponding eigenenergies are ${ }^{10}$

$$
E_{p, k}=\frac{1}{2}\left(\frac{\lambda_{p, k}}{R}\right)^{2} .
$$

To test (5) we use the same basis functions as Brownstein, expanding $\phi$ in (5) in terms of

$$
\psi_{m, n}(x, y)=\sin (m \pi x / R) \sin (n \pi y / R),
$$

with $m, n$ varying from 1 up to a maximum value $M$. These functions automatically satisfy the zero amplitude requirement over the straight lines $x=0, y=0$, and the integral over $S$ in (5) reduces to a line integral over the perimeter of the quadrant.

Typical results for this system are shown in Fig. 1, where we study the second lowest eigenvalue obtained with various basis set dimensions and confining potentials, and we also compare them with the estimates obtained using the stationary method derived by

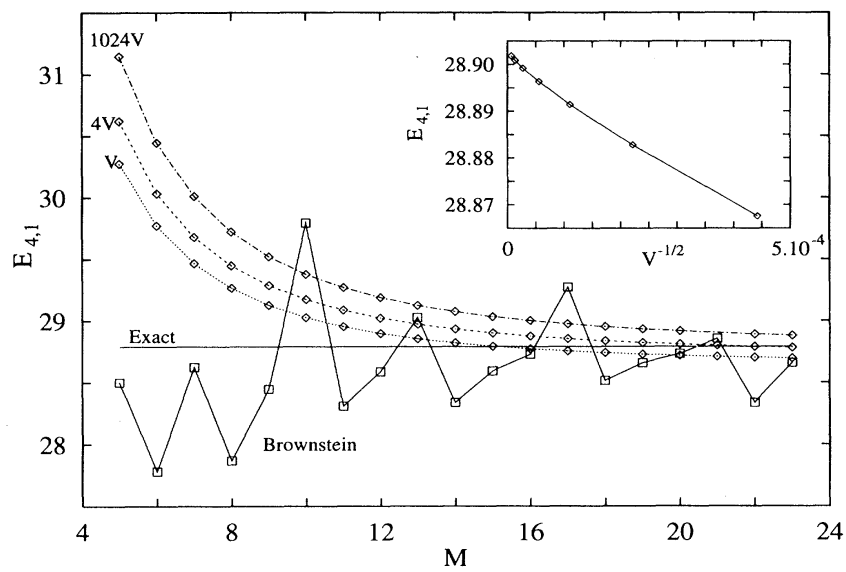

FIG. 1. Variational estimates of the $E_{4,1}$ eigenstate [Eq. (11)] of a two-dimensional free electron confined to a quadrant of radius $R=1$ a.u. Energies evaluated with the embedding method for confining potentials $V, 4 \mathrm{~V}$, and $1024 \mathrm{~V}$, where $V=8 \times 10^{4}$, are compared with those given by Brownstein's method (Ref. 7) as a function of $M$ (see text - basis set size $M^{2}$ ). The inset shows the variation of the eigenvalue given by the embedding method with the confining potential $V$, for the basis set with $M=22$. Similar behavior occurs for smaller basis sets. The exact value of the eigenvalue is $E_{4,1}=28.7915$. 
Brownstein. ${ }^{7}$ Brownstein's method gives a variable number of ghost states below the ground state and so, in this case, we select the eigenvalue closest to the exact answer. We see uniform convergence in the embedding results with an increasing basis set size, unlike the eigenvalues given by Brownstein's method from which it would be impossible to deduce an accurate eigenvalue. Estimates obtained using small confining potentials are better at small basis set sizes due to a cancellation of errors - leakage reduces the eigenvalue, while the variational solution means the result lies above that obtained with a larger basis set - but for large basis sizes, the value converges below the exact eigenvalue. The inset shows the behavior of the eigenvalue with confining potential, from which it is clear that the error due to leakage can be accurately estimated and corrected for.

Even for large basis sets the eigenvalues calculated for this problem retain considerable error. This is due to the choice of basis set, which does not contain sufficient flexibility to satisfy the zero amplitude boundary conditions. A better chosen basis set would greatly improve convergence. This is shown in a second more physical example which was also considered by Brownstein, ${ }^{7}$ and by Gorecki and Byers Brown ${ }^{5}$ and Diamond, Goodfriend and Tsonchev, ${ }^{6}$ where a $\mathrm{H}$ atom is placed 0.5 a.u. off center in a spherical cavity of radius $3 \mathrm{a} . \mathrm{u}$. Our results are presented in Table I along with those of Brownstein, in both cases using the basis functions,

$$
u_{\alpha, \beta}(r, \theta)=e^{-r} r^{\alpha} \cos ^{\beta}(\theta),
$$

where $r$ and $\theta$ are the radial and polar coordinate relative to the atom at the origin, and $\alpha=0,1, \ldots(N-1), \beta=$ $0,1, \ldots(M-1)$. Both methods converge to the same ground state energy with rather few basis functions. The embedding results have been obtained with a confining potential of $V=1.8 \times 10^{9}$ a.u. so that there is negligible error due to leakage. Again they converge uniformly from above, unlike the estimates obtained by Brownstein.

To conclude, we have shown how the embedding method can be used to find the eigenstates of confined
TABLE I. Ground state energy (in a.u.) of a $\mathrm{H}$ atom displaced 0.5 a.u. off center in a spherical cavity, radius 3 a.u. The embedding method results are calculated using Brownstein's basis set (Ref. 7), with $M=N$ so that the number of basis functions is $N^{2} . V$ in Eq. (5) is taken to be $1.8 \times 10^{9}$ a.u. The number in brackets after the eigenvalue is the position of the eigenvalue as ordered by size.

\begin{tabular}{cccc}
\hline \hline Method & $N=2$ & $N=4$ & $N=6$ \\
\hline Embedding & $-0.31730(1)$ & $-0.41323(1)$ & $-0.41389(1)$ \\
Brownstein & $-0.44906(1)$ & $-0.41013(4)$ & $-0.41389(7)$ \\
\hline \hline
\end{tabular}

quantum systems. Like the method due to Brownstein, in the embedding approach there is no need to construct basis functions which implicitly satisfy the boundary conditions - which can be difficult or impossible for complicated geometries - as the boundary condition is imposed as a variational constraint. In contrast to the method due to Brownstein embedding represents a true minimum principle, but, in addition to requiring the evaluation of similar volume integrals, also requires a sometimes tedious surface integral over $S .^{11}$ Interestingly enough, in the case of the Neumann boundary condition on $S$ $\left(\frac{\partial \phi}{\partial n_{S}}=0\right)$, Brownstein's result ${ }^{7}$ is the same as embedding [i.e., (1) with $G_{0}^{-1}=0$ ]. ${ }^{12}$

Finally, we would point out that ideal confinement is invariably a theoretical approximation, and that the embedding method can equally well handle less severe boundary conditions (e.g., those used in Ref. 8).

This paper resulted partially from a collaboration within the European Community Human Capital and Mobility Network " $a b$ initio (from electronic structure) calculation of complex processes in materials" (Contract No. ERBCHRXCT930369). One of the authors (J.E.I.) was supported by FOM (Stichting voor Fundamenteel Onderzoek der Materie).
* Present address: School of Physics, University of Bath, Bath BA2 7AY, United Kingdom.

${ }^{1}$ J.E. Inglesfield, J. Phys. C 14, 3795 (1981).

${ }^{2}$ G.W. Bryant, Phys. Rev. B 29, 6632 (1984)

3 J.W. Brown and H.N. Spector, J. Appl. Phys. 59, 1179 (1986).

${ }^{4}$ P. Csavinszky and H. Oyoko, Phys. Rev. B 43, 9262 (1991).

${ }^{5}$ J. Gorecki and W. Byers Brown, J. Phys. B 22, 2659 (1989).

6 J.J. Diamond, P.L. Goodfriend, and S. Tsonchev, J. Phys. B 24, 3669 (1991).

${ }^{7}$ K.R. Brownstein, Phys. Rev. Lett. 71, 1427 (1993).

${ }^{8}$ J.L. Zhu and X. Chen, Phys. Rev. B 50, 4497 (1994).

${ }^{9}$ M.I. Trioni, J.B.A.N. van Hoof, S. Crampin, G.P. Brivio, and J.E. Inglesfield, Surf. Sci. 307/309, 41 (1994).
${ }^{10}$ The results presented in Table III of Ref. 7 are in Rydberg atomic units, $2 \mathrm{Ry}=1$ Hartree. In Ref. 7 , the factor $(1 / 2)$ in Eq. (20) and the factor 2 in Eq. (24) are incorrect and should be omitted.

${ }^{11}$ Note, however, with regard to Eq. (5), that $-\int u \nabla^{2} v d \tau+$ $\int u \nabla v \cdot d \mathbf{S}=\int(\nabla u) \cdot(\nabla v) d \tau$. This allows the surface integral involving the normal derivative - which will be especially difficult for complicated surfaces - to be combined with the kinetic energy term in $H$ and replaced with a single volume integral. The remaining surface integral only involves the amplitude of the trial function on the surface.

${ }^{12}$ S. Crampin, J.B.A.N. van Hoof, M. Nekovee, and J.E. Inglesfield, J. Phys. Condens. Matter 4, 1475 (1992). 